\title{
Análisis empírico del acceso a cursos virtuales de Literatura Infantil y Programación durante el confinamiento
}

\section{Empirical analysis of access to virtual courses based on children's literature and programming during the lockdown period}

\section{GÉNESIS ANDREÍNA QUISHPE QUINGA D, WENDY CAROLINA CALISPA BOLAGAY*ID, SARA ABIGAIL CUASIALPUD TORO , NELLY CAROLINA ASTUDILLO LARREA (D)}

Universidad de las Fuerzas Armadas ESPE,

Av. General Rumiñahui s/n y Ambato, Sangolquí, Ecuador

wccalispa@espe.edu.ec*

\section{RESUMEN}

A raíz de la crisis sanitaria a causa del COVID-19, la educación cambió de modalidad de presencial a virtual, de manera que el uso de plataformas virtuales se incorporó al estilo de vida cotidiano; durante el período de vacaciones, la Universidad de las Fuerzas Armadas ESPE desarrolló cursos vacacionales virtuales por medio de la plataforma Moodle, de los cuales, el curso de Literatura infantil constó con 503 niños y el curso de Programación tuvo 522 niños inscritos, y se analizó el nivel de frecuencia de acceso de los dos cursos. La metodología utilizada en la investigación es de enfoque cuantitativo, ya que los datos se obtuvieron de los reportes de la plataforma Moodle, donde se verificó el ingreso de los alumnos a los cursos virtuales. Fue a partir del reporte de datos de la plataforma que se evidenció la mayor frecuencia de acceso al curso de programación los días lunes durante el horario de la tarde con un $60,69 \%$. Por otra parte, se observó una mayor frecuencia de acceso los días jueves al curso de literatura con un 50,80 \% en el horario vespertino. Así podemos concluir que, con la creación de los cursos virtuales vacacionales, existió una gran aceptación por parte de los niños, tanto de literatura como de programación, donde el interés más significativo se vio reflejado en los datos obtenidos de la frecuencia de acceso del curso de programación. 
Análisis empírico del acceso a cursos virtuales de Literatura Infantil y Programación durante el confinamiento

Génesis Andreína Quishpe Quinga, Wendy Carolina Calispa Bolagay, Sara Abigail Cuasialpud Toro,

Nelly Carolina Astudillo Larrea • VÍNCULOS-ESPE (2021) VOL.6, No.2: 57-65

Palabras clave: acceso, curso virtual, literatura infantil, programación, plataformas virtuales.

\section{ABSTRACT}

As a result of the health crisis caused by COVID-19, the modality of education changed from face-to-face into virtual, therefore, the use of virtual platforms was incorporated into the daily lifestyle. During the vacation period, the Universidad de las Fuerzas Armadas - ESPE, developed virtual vacation courses through the Moodle platform, from which, the Children's literature course had 503 while the programming one had 522 enrolled children. The level of access frequency was analyzed in both courses. The methodology used in the research is of a quantitative approach since the data was obtained from the reports of the Moodle platform where the students' access to the virtual courses would be verified. It was from the data report of the platform that the highest frequency of access to the Programming course was evidenced on Mondays afternoons with $60.69 \%$. On the other hand, a greater frequency of access to the Literature course was observed on Thursdays afternoons with $50.80 \%$. Thus, we can conclude that with the creation of virtual vacation courses, there was a great acceptance by children, not only for the Literature course, but also for the Programming one, where the most significant interest was reflected in the data obtained from the frequency of access to the Programming course.

Keywords: access, virtual courses, children's literature, programming, virtual platforms.

\section{INTRODUCCIÓN}

González (2015) menciona "El desarrollo de las Tecnologías de Información y Comunicación (TIC) han establecido nuevas aplicaciones didácticas para mejorar el proceso de enseñanza aprendizaje" (p. 6). Mientras la sociedad avanza, el uso de tecnologías está en constante transformación y se vuelve parte del estilo de vida actual, por tal razón, los niños deben adaptarse a las nuevas estrategias propuestas en el aprendizaje virtual.

Las Tecnologías de la Información y Comunicación (TICs) han permitido el surgimiento de los ambientes virtuales formativos entendidos como los espacios o entornos que favorecen el aprendizaje de los alumnos con apoyo en la tecnología, mediante la cual se genera una interacción entre los estudiantes y el docente (Contreras \& Garcés, 2018. p.15). Como consecuencia nacen las plataformas educativas virtuales que han posibilitado ampliar los conocimientos de estudiantes y maestros sin importar tiempo y espacio geográfico. Una de ellas es Moodle, Avello, Rodríguez, Dueñas (2016) la definen como "un sistema de gestión de cursos diseñado para crear y gestionar espacios de aprendizaje online adaptados a las necesidades de profesores, estudiantes y administradores" (p. 3). Actualmente las tecnologías digitales son parte de la vida cotidiana, por lo que los niños ya empiezan a utilizarlas e introducirlas en su formación académica.

En un estudio longitudinal de niños australianos se menciona que el juego digital de hasta 240 minutos por semana se asocia con mejores puntajes en alfabetización y pensamiento matemático en niños de 10 y 11 años. Esta evidencia es pionera en el campo porque es el primer estudio longitudinal realizado de 
una población a nivel nacional. Lo que también encontraron fue que los niveles más bajos de juego (120 minutos) no mostraron ganancias en los logros (Walker, Danby \& Hatzigianni, 2018, p.8). En los cursos propuestos por la Universidad de las Fuerzas Armadas ESPE, los niños tienen la libertad de poder ingresar cuando ellos deseen sin la presión de que se sientan obligados a permanecer por alguna cantidad de tiempo predeterminada. Asimismo, Chaudron \& Kervin (2018) en su estudio de siete países muestra cómo las familias generan reglas de acceso y uso de tecnologías digitales para niños de 6 a 8 años, se identificó que, aunque las familias en general estaban predispuestas positivamente a que sus hijos usaran tecnologías, ellos no se sentían seguros o no tenían los conocimientos necesarios para la selección de aplicaciones y sitios web que apoyen a sus hijos en el uso de tecnologías en el hogar. Por tal motivo es necesario que, para el desarrollo de las actividades en las plataformas, se lo realice con la ayuda de un adulto, y también con un acompañamiento por parte de las organizadoras de los cursos virtuales. Finalmente, en el estudio de EEG (Examen de Electroencefalografía) se afirma que, en los últimos años, el rápido desarrollo de la tecnología de asistencia multimedia y las ayudas didácticas han llevado a la creciente variedad de dispositivos de lectura para niños. El experimento se realizó en junio de 2015 y los participantes fueron 42 estudiantes de primer grado de una escuela primaria en el distrito de Xindian, los resultados mostraron que la concentración de los que leían los libros ilustrados tradicionales era mayor que la de los que leían los libros ilustrados electrónicos (Yeh \& Chen, 2016, p. 1). De tal manera que la forma del libro de imágenes tuvo una influencia significativa en la comprensión de los niños del contenido del cuento.
La presente investigación tiene como objetivo analizar la preferencia de acceso de los niños de 5 a 12 años a los cursos vacacionales virtuales de literatura infantil y de programación en función de los diferentes horarios del día (mañana, tarde y noche).

\section{MATERIALES Y MÉTODOS}

Con la llegada de la pandemia Covid-19 al Ecuador, el sistema educativo tuvo una transformación en la modalidad de estudio en todos los niveles educativos; las clases presenciales pasaron a la modalidad virtual. Por ello, la Universidad de las Fuerzas Armadas ESPE ubicada en Sangolquí-Ecuador, y específicamente el Departamento de Ciencias Humanas y Sociales a través de la carrera de Educación Inicial, programó cursos virtuales vacacionales de literatura infantil y programación. Los cursos fueron implementados en la plataforma educativa Moodle y estaban dirigidos a niños entre edades de 5 a 12 años, estos cursos iniciaron el 19 de agosto y finalizaron el 31 de agosto del 2020. El curso de Literatura Infantil constó de 3 módulos: "Textos literarios", "Conocimiento de nuevas palabras" y "Lectura de cuentos más extensos". Por otra parte, el curso de Programación para niños contempló 4 módulos: el primero de ellos "Conocimiento del mundo de programación", seguido de "Mis primeros pasos en la programación", el tercero "Jugando con la programación" y por último "Más allá de la programación".

La difusión de los cursos vacacionales se realizó a través de la red social Facebook del Departamento y de la institución. La inscripción se realizó por medio de formularios desde el 12 al 16 de agosto del 2020, donde se recopilaron datos informativos 
del usuario. Se obtuvieron para el curso de Literatura Infantil un total de 503 alumnos y en el curso de Programación para niños un total de 522 .

La metodología utilizada en la investigación es de enfoque cuantitativo porque los datos se recopilaron de los reportes generados por la plataforma Moodle, donde se verificó el acceso de los alumnos a los cursos virtuales propuestos. Para la tabulación de los resultados se clasificaron las interacciones analizando los siete días de la semana y se establecieron tres horarios de acceso: de 07h00 a 11h59 de la mañana, 12h00 a 17h59 de la tarde y 18 h00 a 23 h59 de la noche. Cabe recalcar que a partir de las $23 \mathrm{~h} 59$ no se registran ingreso hasta las $07 \mathrm{~h} 00$ de la mañana siguiente. Una vez establecidos los horarios de ingreso correspondiente a los días de la semana, se identificó la frecuencia de acceso de los cursos de literatura infantil y programación. Para la representación de los resultados se realizaron gráficos de la frecuencia de acceso desde el 17 hasta el 29 de agosto del 2020.

\section{RESULTADOS}

Con el análisis de los 1025 niños inscritos en los cursos (522 niños curso de programación y 503 de literatura) se obtuvieron los siguientes resultados mediante unos gráficos de barras.

\section{Figura 1}

Frecuencia de acceso de los participantes horario de la mañana

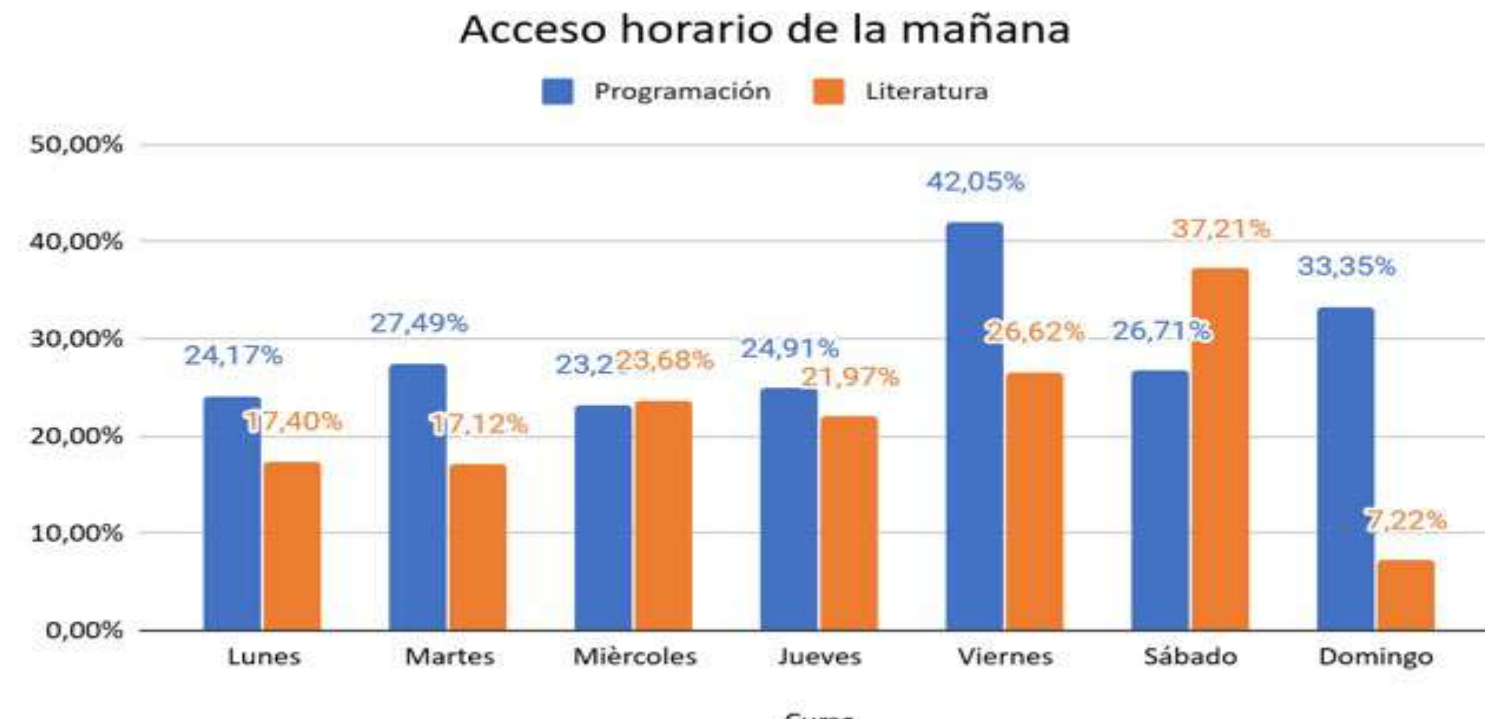

Nota. Análisis de los 1025 niños inscritos en los cursos. 
Como se observa en la Figura 1, la preferencia de acceso es el horario de la mañana de 07h00 a 11h59, en el curso de Programación se evidencia el mayor porcentaje de 42,05\% los días viernes, y el menor porcentaje corresponde al 23, 23\% los días miércoles; mientras que en el curso de Literatura el mayor porcentaje pertenece a los días sábados con un 37,21 $\%$, y el menor porcentaje se encuentra los días domingos con un $7,22 \%$. Se tiene entonces como resultado que el curso de Programación tiene un mayor acceso en el transcurso de la mañana.
Como se observa en la Figura 2, la preferencia de acceso en el horario de la tarde es de $12 \mathrm{~h} 00$ a $17 \mathrm{~h} 59$; en el curso de Programación se evidencia un mayor porcentaje de 60,69\% los días lunes, y el menor porcentaje corresponde al 47,51\% los días viernes. En el curso de Literatura el mayor porcentaje pertenece a los días jueves con un 50,80 \%, y el menor porcentaje se encuentra los días sábados con un 38,55\%. De manera que, en el horario de la tarde, el mayor acceso de participantes lo tiene el curso de Programación.

\section{Figura 2}

Frecuencia de acceso de los participantes horario de la tarde

\section{Acceso horario de la tarde}

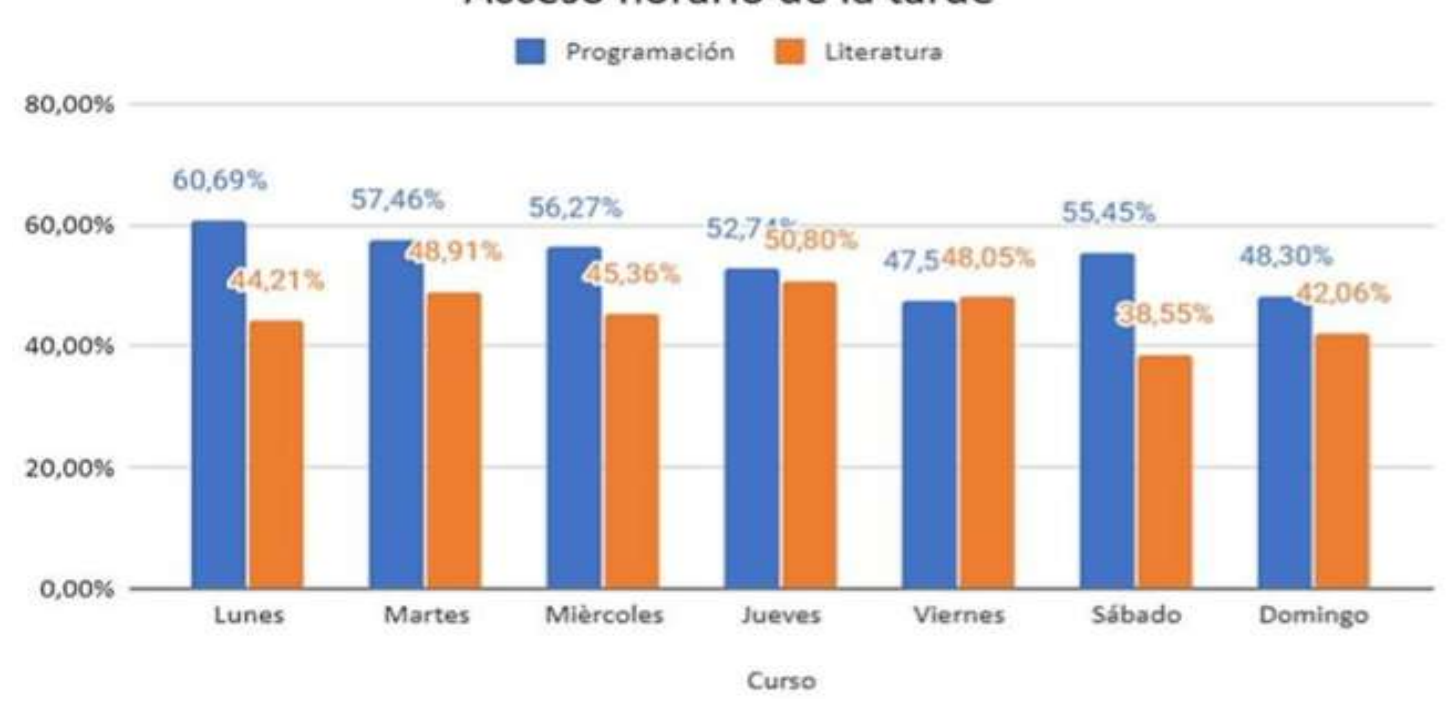

Nota. Análisis de los 1025 niños inscritos en los cursos. 
Análisis empírico del acceso a cursos virtuales de Literatura Infantil y Programación durante el confinamiento

Génesis Andreína Quishpe Quinga, Wendy Carolina Calispa Bolagay, Sara Abigail Cuasialpud Toro,

Nelly Carolina Astudillo Larrea • VÍNCULOS-ESPE (2021) VOL.6, No.2: 57-65

Como se observa en la Figura 3, la preferencia de acceso en el horario de la noche de $18 \mathrm{~h} 00$ a $23 \mathrm{~h} 59$, en el curso de Programación se evidencia el mayor porcentaje de 22,35 $\%$ el día jueves y donde el menor porcentaje corresponde al 10,43\% los días viernes, mientras que en el curso de
Literatura el mayor porcentaje pertenece al día domingo con un $50,72 \%$, y el menor porcentaje se ubica el día sábado con un $24,24 \%$. De manera que, en el horario de la noche, el mayor acceso de participantes se encuentra en el curso de Literatura.

\section{Figura 3}

Frecuencia de acceso de los participantes horario de la noche

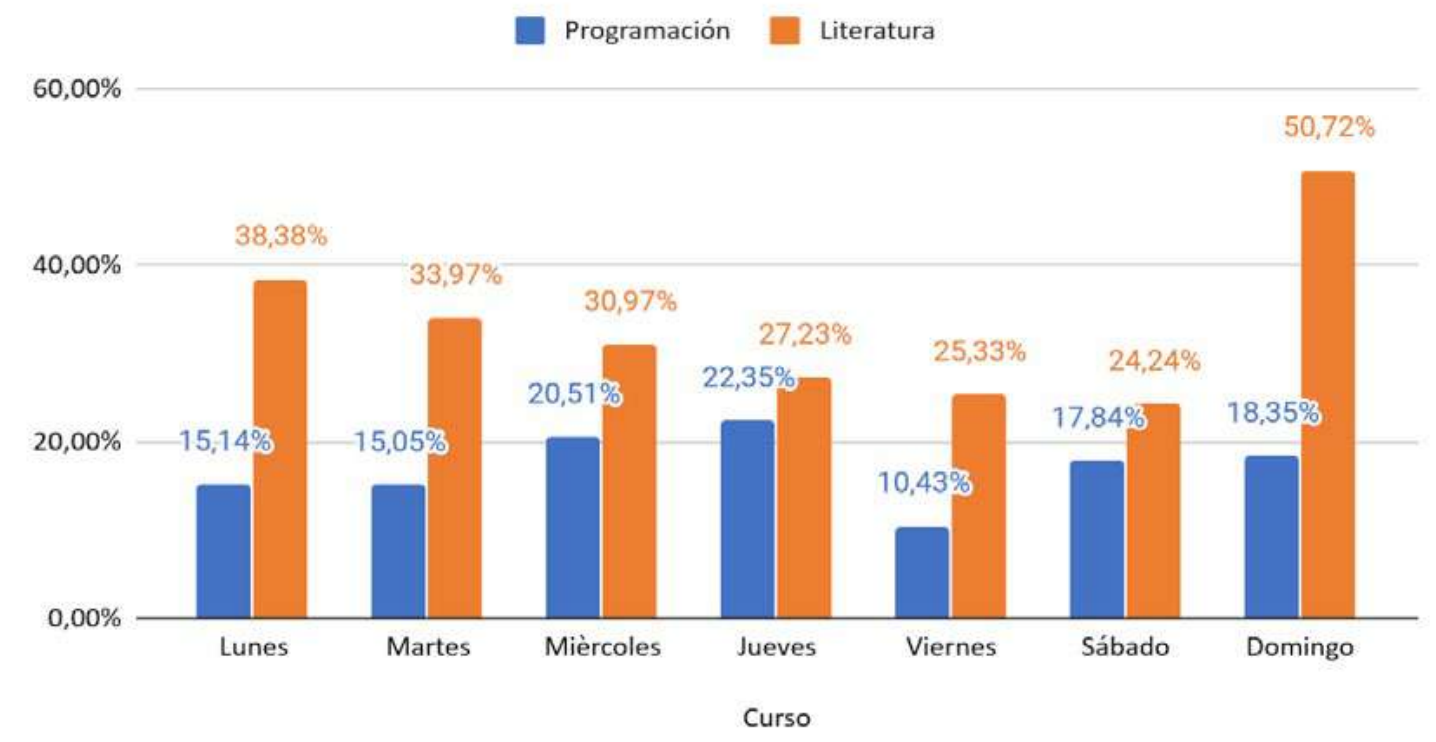

Nota. Análisis de los 1025 niños inscritos en los cursos. 


\section{DISCUSIÓN}

El objetivo de la investigación es analizar la frecuencia de acceso de los niños a los cursos vacacionales virtuales de programación y literatura, el estudio es de enfoque cuantitativo. El análisis estadístico del curso de programación evidenció que su mayor grado de frecuencia de acceso fueron los días jueves en el horario de la tarde y el menor ingreso que se registró fue los días viernes en el horario de la noche. En tanto que en el curso de literatura se logró visualizar que su mayor grado de asistencia fue los días jueves en el horario de la tarde, mientras que el menor grado de asistencia se ubicó el día domingo en el horario de la mañana.

A partir de la interpretación de los datos recopilados se evidenció una mayor frecuencia de ingreso al curso de Programación, la preferencia por el curso virtual podría deberse a que los niños al estar en edades menores a los 13 años sienten más curiosidad sobre el tema de programación. En el estudio sobre la motivación y el interés por la programación, se afirma que, aunque las diferencias son muy sutiles, los casos de apatía o desinterés son más frecuentes en grupos de estudiantes que en promedio tienen más de 13 años o que han iniciado la adolescencia. Los alumnos de entre 10 y 13 años se pueden motivar más fácilmente y también son capaces de mantener un nivel de interés constante durante el taller (Herrera, 2018, p. 35). De manera que los cursos de Programación y Literatura Infantil. al ser dirigidos a niños en edades comprendidas entre 5 y 12 años, concuerda con lo mencionado anteriormente, donde los niños prefirieron acceder mayor cantidad de veces a realizar las actividades propuestas en el curso de Programación. De igual forma, el curso de Programación impartió conocimientos propios de cada tema que se abarca durante este proceso de enseñanza-aprendizaje, además que propicia una estimulación de destrezas que van adquiriendo en la realización de las diferentes actividades.

Entre las habilidades que se pudieron observar en KODU, algunas preguntas y sus respuestas obtenidas por los docentes y acompañantes del proyecto, se encontró que en cada proceso se detectaron líderes naturales, puesto que en muchos casos algunos niños tomaban la iniciativa antes de que se les dieran algunos parámetros. Al utilizar la programación, los niños se convierten en generadores de ideas, por lo tanto, en cada actividad siempre plantearon diversas soluciones (Suárez, 2015, p. 47). Por lo que estos resultados demuestran los avances cognitivos que se logran a través de estos cursos de programación, mismos que ayudan a un mejor desenvolvimiento académico, como también a nivel personal y social, motivo por el cual el curso de Programación tiene relevancia y una mayor cantidad de acceso que les ayuda a despertar un interés por la tecnología.

\section{CONCLUSIONES}

En el curso de Literatura el mayor ingreso visible fue los días jueves en el horario de la tarde de $12 \mathrm{~h} 00$ a 17 h59 con un 50,80 $\%$, mientras que el menor porcentaje de los tres horarios del día (mañana, tarde y noche) fue de 7,22\% el día domingo en el horario de la mañana de 07h00 a 11 h59.

A partir de los datos recopilados de la plataforma Moodle en el curso de Programación se observó que el mayor ingreso de los 
niños fue en el horario de la tarde de 12 h00 a 17 h59 los días lunes con el mayor porcentaje de 60,69\%.

Se concluye que, en los cursos, tanto de Literatura como de Programación, se obtuvo una gran aceptación por parte de los niños en los diferentes horarios del día (mañana, tarde y noche). Esto se le puede atribuir al interés y motivación de los juegos en cada módulo.

\section{AGRADECIMIENTOS}

A la Universidad de las Fuerzas Armadas ESPE, al director del Departamento de Ciencias Humanas y Sociales y a las organizadoras de los cursos virtuales, con la creación de estos cursos que permitió llegar a un aprendizaje significativo a los niños mediante actividades lúdicas. Asimismo, a los docentes que formaron parte del desarrollo del presente trabajo y que brindaron una orientación idónea.

\section{REFERENCIAS}

Avello Martínez, R., Rodríguez Muñoz, R. Dueñas Figueredo, J. O. (2016). Una experiencia con Moodle y herramientas Web 2.0 en el postgrado. Universidad y Sociedad [seriada en línea], 8 (4). pp. 58-64. http://rus.ucf.edu.cu/

Cedeño, E. L., \& Murillo, J. A. (2019). Entornos virtuales de aprendizaje y su rol innovador en el proceso de enseñanza.
Revista de Ciencias Humanísticas y Sociales, Vol. 4, No 1. p. 1 19-127. https://revistas.utm.edu.ec/index.php/Rehuso/article/ view/2156/2275

Contreras, A. F., \& Garcés, L. M. (10 de noviembre de 2018). Ambientes Virtuales de Aprendizaje: dificultades de uso en los estudiantes de cuarto grado de primaria. SCIELO. http://www.scielo.org.co/ $\mathrm{pdf} / \mathrm{prsp} / \mathrm{n} 27 / 2389-993 X-p r s p-27-00215 . p d f$

Danby, S. J., Fleer, M., Davidson, C., \& Hatzigianni, M. (2018). Digital Childhoods Across Contexts and Countries. In International Perspectives on Early Childhood Education and Development (Vol. 22, pp. 1-14). Springer. Recuperado de: https://doi. org/10.1007/978-981-10-6484-5 1

González, M. (2015). Enriquecimiento tecnológico y psicopedagógico del concepto de comunidades de práctica en la educación a distancia. WorldWideScience. https://revistas.um.es/red/article/ view/242421/184241

Herrera, A. (2018). Effects on the School Performance of Teaching Programming in Elementary and Secondary Schools. Springer Nature Switzerland. https://doi.org/10.1007/978-3-03002750-6 3

Suárez Castillón, S. A., \& Soto Arévalo, F. S. (2015). Evaluación cualitativa de la utilización del lenguaje de programación visual kodu en niños de educación básica. Revista Tecnura, 19 (46), 37. https://doi.org/10.14483/udistrital.jour.tecnura.2015.4.a03

Yeh, T.-L., \& Chen, C.-C. (2016). A Study of e-Picture Books and Traditional Picture Books on Children's Reading Attention. https://docs.google.com/document/d/160dCMHCSncjDPo8cZ ZTthONq8wb0crFqGOmutAQIFDQ/edit 


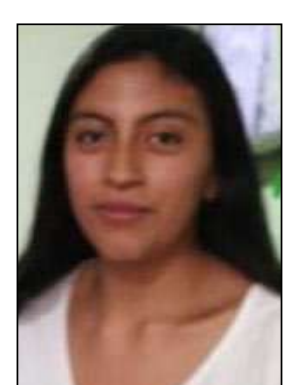

\section{WENDY CAROLINA} CALISPA BOLAGAY

Actualmente vive en el Valle de los Chillos en la cuidad de Sangolquí, está cursando el tercer semestre de la Carrera de Educación Inicial en la Universidad de las Fuerzas Armadas ESPE.

Está siguiendo un curso de la misma Universidad llamado Gamifica tu aula con el objetivo de conocer las diferentes herramientas para brindar una enseñanza de forma dinámica a los niños.

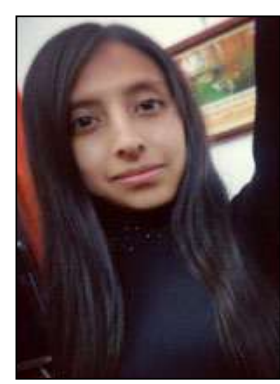

SARA ABIGAIL CUASIALPUD TORO

Nació en Tulcán, es estudiante de tercer semestre de la Universidad de las Fuerzas Armadas ESPE, en la Carrera de Educación Inicial, su visión de enseñar es que debemos educar a los niños con amor y paciencia ya que son seres diferentes, es así que educar es el primer paso para un cambio verdadero en la sociedad.

Dedicada al estudio y la música.

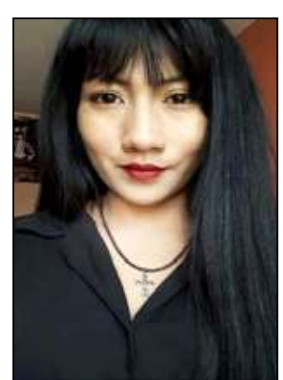

\section{GÉNESIS ANDREÍNA} QUISHPE QUINGA

Actualmente vive en la ciudad de Quito, está cursando el tercer semestre de la Carrera de Educación Inicial en la Universidad de las Fuerzas Armadas ESPE, pertenece al Ensamble de Cuerdas de la Universidad.

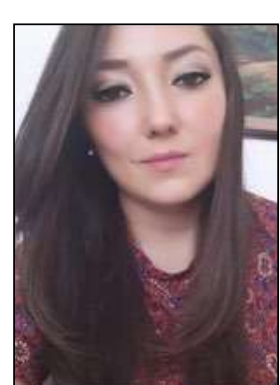

\section{MSC. NELLY CAROLINA LARREA ASTUDILLO}

Graduada de la Maestría en Utilización Pedagógica de las Tecnologías de Información y Comunicación en el Instituto Politécnico de Leiria-Portugal. Graduada en la Universidad de las Fuerzas Armadas ESPE, obteniendo título de Licenciada en Ciencias de la Educación, mención Educación Infantil. Actualmente es docente del Departamento de Ciencias Humanas y Sociales de la Universidad de las Fuerzas Armadas ESPE. 